\title{
PHYSICAL ACTIVITY AND HEALTH RELATED QUALITY OF LIFE OF BREAST CANCER WOMEN SUBMITTED TO NEOADJUVANT CHEMOTHERAPY
}

Julia de M. R. Medina1', Suzana S. de Aguiar', Luiz Claudio S. Thuler ${ }^{1}$, Marcelo Adeodato Bello1, Anke Bergmann ${ }^{1}$ Hospital do Câncer III, Instituto Nacional de Câncer - Rio de Janeiro (RJ), Brazil.

Objective: To evaluate the influence of the level of Physical Activity (PA) in the Health Related Quality of Life (HRQOL) of women submitted to neoadjuvant chemotherapy for the treatment of breast cancer (BC). Methods: Prospective cohort study in women diagnosed with BC, with neoadjuvant chemotherapy with adriamycin and cyclophosphamide (AC), from April 4, 2016 to August 2, 2017, at Hospital do Cancer III (HCIII / INCA). The patients were submitted to the interview, physical examination and application of questionnaires at the first consultation with the oncologist and after 21 days of the end of the 4th cycle of neoadjuvant chemotherapy with AC. This project was submitted and approved by the Research Ethics Committee (CAAE 51100615.7.0000.5274). The analysis of the population and the simple and adjusted linear regression analysis between the levels of PA and the domains of HRQoL were performed, considering the $95 \%$ confidence interval. Results: A total of 253 women were included, with a mean age of 50.87 years ( \pm SD 10.62). After adjusting, the women with higher levels of PA have a better overall QoL (Beta 4.06, p=0.040) after neoadjuvant chemotherapy. There was no statistically significant difference between the AF levels after a neoadjuvant chemotherapy and the HRQoL domains. Conclusion: Patients who had higher levels of pre-diagnosis PA had better overall QoL after neoadjuvant chemotherapy and there was no difference between groups when assessing the levels of PA and HRQoL after the AC neoadjuvant chemotherapy regimen. 